\title{
Low-fat high-carbohydrate diet and plasma sex hormones
}

Sir

The paper of Boyd et al (1997) (Br J Cancer 76: 127-135) reports the results of randomization of 220 premenopausal women to a low-fat intake (21.2\% energy from fat) or to a control diet $(33.1 \%$ energy from fat) for 2 years: this is both the largest and the longest duration controlled trial that has been published and the results are therefore of great interest. At the end of the trial. serum concentrations of oestradiol and progesterone were significantly lower in the intervention group than in the control group (by 20\% and 35\% respectively). although the differences between groups in the changes in these hormones during the trial were small and were not statistically significant. The authors pointed out that the differences between groups at the end of the trial appeared to be due to long menstrual cycles in a small number of subjects in the intervention group and suggested that the low-fat diet may have accelerated the onset of menopausal changes. This would be a very important finding. but some further details would be helpful in interpreting the results.

One possibility that should be checked is whether. by chance. a higher proportion of the intervention group than of the control group was entering menopause at the beginning of the trial. This is a possibility because there were $50.9 \%$ of women in the intervention group but only $43.3 \%$ of women in the control group aged $45+$ years at baseline and because median baseline FSH was $18 \%$ higher in the intervention group $(P=0.08)$. Did any subjects have baseline FSH concentrations suggestive of the onset of menopause
(>30 IU $\left.1^{-1}\right)$ ? Some information on the date of the last menstrual period was collected at baseline: this could not be used to interpret the values of the baseline hormone assays. but it might provide some information on whether any of the subjects had very long menstrual cycles at baseline and whether this differed between groups. Could the authors provide a table of days since last menstrual period at baseline by dietary group?

Figure 1 in the paper shows that. at the end of the trial. there were eight women in the intervention group but only two women in the control group who reported that it was more than 50 days since their last menstrual period. It would be useful to know the ages of these ten women and their FSH values at baseline and at 2 years (these can only be roughly estimated from the Figure). It might also be interesting to divide the data by age to see whether the effect is concentrated at ages 45 years and above and whether there is any difference between groups below age 45 years.

The authors state that the difference in dietary fibre intake between the intervention and control groups at 2 years was statistically significant. which could imply that the hormonal differences were influenced by the change in fibre intake. However. the difference in fibre intakes was small. and the data in Table 2 indicate that it was not statistically significant.

TJ Key

Imperial Cancer Research Fund. Cancer Epidemiology Unit. Gibson Building. Radcliffe Infirman: Oxford OX2 6HE. UK

\section{Low-fat high-carbohydrate diet and plasma sex hormones - reply}

\section{Sir}

We thank your correspondent for their letter and reply to the questions in the order in which they were posed.

1. The number of subjects with baseline FSH levels greater than $30 \mathrm{IU} \mathrm{L}^{-1}$ was nine for the intervention group (I) and 11 for the controls $(\mathrm{C}$ ). The mean values of FSH for those with values over 30 . were $52.8 \mathrm{IU} \mathrm{L}^{-1}(\mathrm{I})$ and $57.4 \mathrm{IU} \mathrm{L}^{-1}(\mathrm{C})$.

2. The number of days from the last menstrual period at baseline is not available. We did. however. collect information about maximum cycle length. For the intervention group (I) and for controls ( $\mathrm{C}$ ) the maximum cycle length was as follows:

\begin{tabular}{lll}
\hline & I & C \\
\hline Mean & 29.3 & 28.5 \\
Median & 28.0 & 28.0 \\
Interquartile range & $28-30$ & $27.5-30$ \\
\hline
\end{tabular}

3. For the ten women who reported more than $\mathbf{5 0}$ days since last period at 2 years. their ages and FSH values [mean (median)] were:

\begin{tabular}{llccc}
\hline Group & $\boldsymbol{n}$ & Age (years) & FSH baseline & FSH 2 year \\
\hline $\mathrm{I}$ & 8 & $47.4(49)$ & $25.6(22)$ & $134.9(150.5)$ \\
$\mathrm{C}$ & 2 & $45(45)$ & $41(41)$ & $47(47)$ \\
\hline
\end{tabular}

4. Division of the data at age 45 years showed similar findings to those shown in Table 3 of our paper. In women under age 45 years. the median oestradiol level in the intervention group ( $n$ = 55) was 306 [interquartile range (IQR) 183-514] at baseline and 275 (IQR $134-450)$ at 2 years. In controls $(n=59)$ the values were 274 (IQR $165-438$ ) at baseline and 352 (IQR $172-450$ ) at 2 years. The difference in median levels at 2 years was not statistically significant $(P=0.41)$. In women aged over 45 years at entry. the median oestradiol level in the intervention group ( $n=57$ ) was 259 (IQR 191-445) at baseline and 
270 (IQR 96-457) at 2 years. In controls $(n=45)$ the values were $367(230-475)$ at baseline and 345 (IQR 245-455) at 2 years. The difference in median levels at 2 years was statistically significant $(P=0.03)$.

5. The difference in fibre intake shown in Table 2 is not statistically significant. However. in the trial as a whole. when the difference in fibre intake is the same but the number of subjects is of course much larger. the difference between intervention and control groups is highly significant.
We thus find no evidence to support the suggestion that a higher proportion of the intervention group was entering menopause at baseline. However. it does seem that the main effect of the dietary intervention on oestradiol levels may be in women over 45 years. although this conclusion is based on small numbers and is obviously preliminary.

N Boyd

Division of Epidemiology and Statistics. Ontario Cancer Institute. Princess Margaret Hospital. Toronto. Ontario. Canada

\section{Basal cell carcinoma of the face: surgery or radiotherapy? Results of a randomized study}

Sir,

I read with interest the recent paper by Avril et al (1997) describing the results of a prospective study comparing surgery and radiotherapy in the management of basal cell carcinomas (BCCs) of the face in 347 patients. Because both tumour recurrence rates and cosmesis were found to be significantly better in the surgically managed group. the authors concluded that surgery should be considered as the first-line treatment of choice for facial BCCs less than $4 \mathrm{~cm}$ in diameter.

While there is undoubtedly a need for good randomized prospective trials comparing the effectiveness of the two treatment options. this study did not address the issues of management as they are commonly encountered in current clinical practice. In the study. the authors compared the treatment modalities in patients with a wide range of clinical presentations. The size of tumours. for example. ranged from $3-5 \mathrm{~mm}(10 \%)$ to $31-10 \mathrm{~mm}(0.9 \%)$ in diameter. with $57 \%$ of tumours $<10 \mathrm{~mm}$ and $93 \%<20 \mathrm{~mm}$. Most lesions were non-ulcerated nodules clinically. with a smaller number of superficial $(22 \%)$ and morphoeic-type BCCs $(4 \%)$. The affected sites included the forehead. cheeks. chin. ears and nose.

It is perhaps these broad inclusion criteria that weakened the overall value of the trial. One of the fundamental principles of skin cancer management is. when possible. to completely remove tumours (Fleming et al. 1995). In practice. most dermatologists would opt for excision of lesions if it were both technically possible and likely to give good cosmetic results. As the majority of BCCs in both treatment groups were $<1 \mathrm{~cm}$ in diameter. it seems likely that many of these tumours would have been amenable to simple surgical excision with primary closure. In those cases treated with radiotherapy. surgical intervention would not only have avoided multiple outpatient visits. or even lengthy inpatient stays. but would have permitted histological assessment of resection margins.

In their conclusions. the authors stated that. for facial BCCs less than $4 \mathrm{~cm}$ in diameter. surgery is the treatment of choice. It is well recognized that radiotherapy has a valuable role in the management of particular clinical problems. such as tumours affecting cartilaginous areas and the treatment of large ulcerated lesions often encountered in elderly patients (Fleming et al. 1995). This conclusion. while undoubtedly true for small lesions amenable to excision. disregards the usefulness of radiotherapy in the management of larger and more awkwardly situated lesions.

Dr Susan Holmes

Department of Dermatolog:

University of Glasgow.

\section{REFERENCES}

Avril MF. Auperin A. Margulis A et al 1 1997) Basal cell carcinoma of the face: surgen or radiotherapy? Results of a randomized study. $\mathrm{Br} J$ Cancer $\mathbf{7 6}$ : $100-106$

Fleming ID. Amonette R. Monaghan T and Fleming MD (1995) Principles of management of basal and squamous cell carcinoma of the skin. Cancer 75 : $699-704$

\section{Expression of bch-2 protein in follicular lymphomas: a report from a south Indian hospital}

Sir,

The $\mathrm{t}(14: 18)$ translocation juxtaposes part of the immunoglobulin heavy-chain gene on chromosome 14 with the bcl-2 gene on chromosome 18. This translocation was discovered in most follicularcentre cell lymphoma. The prevalance of $t(14: 18)$ shows a geographical predilection. being highest in the USA and lowest in Japan (Isaacson. 1991). The bcl-2 oncoprotein can be easily localized using immunohistochemical staining. and this has been studied in follicular lymphomas occurring in the West. We assessed the frequency of $b c l-2$ expression in follicular lymphomas in 51 subjects from the Indian subcontinent.

Out of a total of 406 cases of non-Hodgkin's lymphoma diagnosed from 1 March 1995 to 30 September 1997. 55 were follicular lymphoma ( $13.5 \%$ ). Formalin-fixed paraffin-embedded tissue samples for immunohistochemistry were available in only 51 of these cases. Immunohistochemical analysis was performed on 